\title{
Influência da redução medicamentosa da pressão intra-ocular na medida da espessura da camada de fibras nervosas da retina de olhos hipertensos e glaucomatosos pela polarimetria de varredura a laser
}

\author{
The influence of intraocular pressure reduction with medication on retinal nerve \\ fiber layer thickness measurements obtained with scanning laser polarimetry \\ inglaucomatous and hypertensive eyes
}

Rodrigo Rezende Gomes Avelino ${ }^{1}$ Pedro Alexandre Henriques Luis ${ }^{2}$ Márcia Medeiros $^{3}$

Vital Paulino Costa ${ }^{4}$
Pós-graduando e Médico assistente do Setor de Glaucoma do Hospital de Clínicas da Universidade Estadual de Campinas - UNICAMP - Campinas (SP) - Brasil. Médico assistente da Universidade de Taubaté - (SP) Brasil.

Fellow do Setor de Glaucoma do Hospital de Clínicas da UNICAMP - Campinas (SP) - Brasil.

Professor Livre Docente pela Faculdade de Medicina de Universidade de São Paulo - USP - São Paulo (SP) - Brasil, Chefe do Setor de Glaucoma do Hospital de Clínicas da UNICAMP - Campinas (SP) - Brasil.

Endereço para correspondência: Rodrigo Rezende Gomes Avelino. Rua Dr. Paulo Castro Pupo Nogueira, 21 Campinas (SP) CEP 13015-300

E-mail: rodrigoavelino@uol.com.br

Recebido para publicação em 27.07.2005

Última versão recebida 26.01 .2006

Aprovação em 12.02.2006

\section{RESUMO}

Objetivo: Avaliar o efeito da redução da pressão intra-ocular (PIO) obtido com o uso de terapia medicamentosa na espessura da camada de fibras nervosas da retina medida pela polarimetria de varredura a laser (PVL) em pacientes glaucomatosos ou hipertensos oculares. Métodos: Trinta e sete olhos de 37 pacientes foram prospectivamente incluídos no estudo e avaliados com a PVL sem uso de medicação ocular hipotensora e num período entre 15 e 30 dias após a instituição de medicação ocular hipotensora, que resultou em redução da PIO de pelo menos $25 \%$. Os parâmetros medidos pela PVL antes e após a redução da PIO foram comparados com o teste t de Student pareado. Resultados: A PIO média dos 37 pacientes diminuiu significativamente de $26,57 \pm 4,23 \mathrm{mmHg}$ para $16,54 \pm 2,92 \mathrm{mmHg}(\mathrm{p}<0,05)$ após terapia medicamentosa. Não houve diferença estatisticamente significativa entre os valores dos 10 parâmetros do PVL medidos antes e após a administração de medicação ocular hipotensora ( $p>0,05)$. Conclusão: A redução da PIO com o uso de medicação ocular hipotensora não altera a medida da espessura da camada de fibras nervosas da retina pela PVL em pacientes com glaucoma ou hipertensão ocular.

Descritores: Glaucoma; Pressão intra-ocular/quimioterapia; Fibras nervosas/patologia; Retina/patologia; Técnicas de diagnóstico oftalmológico; Lasers/uso diagnóstico

\section{INTRODUCÃO}

O glaucoma é uma neuropatia óptica crônica progressiva, caracterizada por alterações típicas do disco óptico e da retina com repercussões características no campo visual ${ }^{(1)}$. A perda de fibras nervosas da retina precede as alterações de campo visual. Existem evidências de que aproximadamente $35-50 \%$ das fibras nervosas da retina podem ser perdidas em um olho glaucomatoso com perimetria acromática ainda normal ${ }^{(2)}$. No entanto, a avaliação clínica do disco óptico e da camada de fibras nervosas da retina (CFNR) é qualitativa e subjetiva, com grande variabilidade intra e inter-observador ${ }^{(3-4)}$.

Por esse motivo, novos métodos objetivos foram desenvolvidos para permitir a avaliação quantitativa do disco óptico e da CFNR. Como o dano ao disco óptico e à CFNR precede a lesão de campo visual, estes métodos 
podem ajudar o oftalmologista no diagnóstico precoce e monitoramento do glaucoma.

A PVL (GDx - Nerve Fiber Analyzer; Laser Diagnostic Technologies, San Diego, EUA) é um desses métodos. O GDx usa a tecnologia de varredura a laser acoplado a um modulador de polarização. Baseia-se na propriedade birrefringente da CFNR, que gera um retardo em um feixe de laser polarizado quando este a atravessa. Este retardo é proporcional à espessura da $\mathrm{CFNR}^{(5)}$. O GDx pode ser usado tanto no diagnóstico do glaucoma inicial ${ }^{(6)}$, quanto para detectar e quantificar progressão, mesmo que esta não seja evidente com a perimetria ${ }^{(7)}$.

A terapia atual do glaucoma para impedir a progressão da doença consiste em reduzir a PIO por meio de medicações, laser ou cirurgia ${ }^{(8)}$. No entanto, existem diversos estudos mostrando que a diminuição da PIO pode provocar alterações na topografia do disco óptico medida com o oftalmoscópio confocal de varredura a laser, mesmo quando esta redução é obtida com tratamento medicamentoso ${ }^{(9)}$. Do ponto de vista prático, isto representa uma importante limitação para a análise de progressão do dano glaucomatoso por meio desta tecnologia. Por outro lado, não existem na literatura estudos que avaliem a repercussão da queda da PIO obtida com medicação sobre as medidas da CFNR obtidas com o PVL.

O objetivo deste estudo é avaliar o efeito da redução da PIO obtido com o uso de terapia medicamentosa na espessura da CFNR medida pelo PVL em pacientes glaucomatosos ou hipertensos oculares.

\section{MÉTODOS}

Foram prospectivamente incluídos no estudo pacientes com glaucoma ou hipertensão ocular seguidos no Serviço de Glaucoma do Hospital de Clínicas da Unicamp. Um olho de cada paciente foi selecionado aleatoriamente para o estudo quando ambos os olhos respeitavam os critérios de inclusão e exclusão.

Para inclusão no estudo, os pacientes deveriam satisfazer os seguintes critérios: a) Hipertensos oculares: $\mathrm{PIO} \geq 24 \mathrm{mmHg}$ sem medicação às 10 horas, sem anormalidade de disco óptico ou de campo visual. Definiu-se como campo visual normal aquele com GHT normal, valores de MD e CPSD presentes em mais de $95 \%$ da população normal; b) Glaucoma: $\mathrm{PIO} \geq 21 \mathrm{mmHg}$ sem medicação às 10 horas, com anormalidade de disco óptico característica de glaucoma. Definiu-se como anormalidade de disco óptico a presença de pelo menos duas das seguintes alterações: escavação vertical > 0,7, chanfradura do disco óptico, hemorragia peripapilar, escavação nasal, assimetria de escavação > 0,2 e desnudamento do vaso circunlinear.

Pacientes com qualquer das seguintes características foram excluídos: a) idade inferior a 18 anos ou superior a 80 ; b) acuidade visual $\leq 20 / 40 ;$ c) presença de alterações corneanas tais como edema, opacidades, irregularidades ou transplante de córnea; d) qualquer anormalidade que impedisse a realização da tonometria; e) vigência de uveíte anterior; f) catarata nuclear $\geq 3$ na escala de opacidade nuclear ou catarata cortical $\geq 2$ na escala de opacidade cortical ou catarata subcapsular $\geq 0,5$ na escala de subcapsular posterior (LOCS III) ${ }^{(10)}$; g) opacidades vítreas; h) atrofia peripapilar; i) presença de alteração retiniana que pudesse influir nas medidas do LCP; j) incapacidade de manter a fixação ou de colaborar com o exame; k) miopia superior a 5,00DE, hipermetropia superior a $3,00 \mathrm{DE}$ ou astigmatismo superior a 2,50DC; 1 ) impossibilidade de comparecer aos retornos nos prazos estabelecidos.

Os pacientes foram informados sobre o teor do estudo, aceitaram participar e assinaram o termo de consentimento. $\mathrm{O}$ estudo foi aprovado pelo Comitê de Ética da Faculdade Ciências Médicas da Unicamp. Os pacientes foram submetidos à entrevista a fim de relatarem história ocular, tratamento prévio para glaucoma, uso de medicação ocular ou sistêmica, antecedente de doenças oculares ou sistêmicas e antecedente familiar de glaucoma em parentes de até 3 o grau.

O exame oftalmológico constituiu-se de avaliação da acuidade visual com a tabela de Snellen, refração dinâmica subjetiva, tonometria de aplanação com tonômetro de Goldmann, gonioscopia com lente de Posner, biomicroscopia de segmento anterior e biomicroscopia de fundo de olho sob midríase com lente 66D. Os pacientes foram então submetidos à perimetria computadorizada com perímetro Humphrey (programa 24-2, estratégia SITA ou Full Threshold) e avaliação com PVL.

$\mathrm{O}$ exame com PVL foi realizado com o paciente sentado e com a cabeça ereta. As luzes ambientes permaneceram acesas. Não foram obtidas imagens de olhos com pupilas dilatadas. Uma imagem média formada a partir de 3 imagens de boa qualidade foi utilizada para análise dos parâmetros do PVL. Cada imagem resultante consiste de 256X256 pixels, com cada pixel correspondendo ao valor de retardo na sua localização. As imagens obtidas com o PVL são divididas em quadrantes, assim definidos: temporal $\left(334^{\circ}-24^{\circ}\right)$, superior $\left(25^{\circ}-144^{\circ}\right)$, nasal $\left(145^{\circ}-214^{\circ}\right)$, e inferior $\left(215^{\circ}-334^{\circ}\right)^{(11)}$. Os parâmetros do PVL avaliados foram: integral polar total, integral superior, integral temporal, integral inferior, integral nasal, média polar total, média superior, média temporal, média inferior e média nasal. Todos estes parâmetros são medidos numa elipse de 10 pixels de largura e que se situa a 1,75 diâmetros de disco da margem do disco óptico.

Após realização dos exames, prescreveu-se uma medicação ocular hipotensora, a critério do oftalmologista. Num período que variou entre 2 semanas e um mês após a introdução da medicação, o paciente retornou para reavaliação. Se a PIO medida às 10 horas ( \pm 1 hora) apresentasse redução de pelo menos $25 \%$ em relação ao valor inicial, o paciente era submetido à nova avaliação com PVL. Em caso negativo, a medicação inicial era substituída ou uma segunda medicação era prescrita. O paciente retornava, então, dentro de uma semana a um mês para nova avaliação da PIO. Em caso de queda superior ou igual a $25 \%$ em relação ao valor sem medicação, uma nova avaliação com PVL era realizada.

As variações dos parâmetros do PVL e da PIO após uso de medicação ocular hipotensora foram analisadas com o teste $\mathrm{t}$ de Student pareado. Valores de $\mathrm{p}<0,05$ foram considerados estatisticamente significantes. 


\section{RESULTADOS}

Dos 43 pacientes inicialmente selecionados, dois (4\%) não compareceram à segunda consulta. Quarenta e um (96\%) completaram as avaliações, dos quais 37 apresentaram diminuição da PIO de pelo menos $25 \%$ em relação ao valor basal. Assim, para efeito de análise estatística, foram considerados somente os 37 olhos de 37 pacientes que completaram a segunda avaliação e que apresentaram diminuição da PIO de pelo menos $25 \%$. Destes, 24 (65\%) eram do sexo feminino e $13(35 \%)$ do sexo masculino. A média de idade foi $56,7 \pm 13,8$ anos (variando entre 21 e 80 anos). Foram incluídos 24 olhos direitos e 13 olhos esquerdos. Dos 37 pacientes incluídos, 15 (40\%) apresentavam glaucoma e $22(60 \%)$ apresentavam hipertensão ocular.

Entre os olhos incluídos no estudo, 31 (83\%) apresentavam campo visual com MD > - $6 \mathrm{~dB}, 4$ (11\%) mostravam MD entre -6 e $-15 \mathrm{~dB}$ e $1(6 \%)$ apresentava $\mathrm{MD}<-15 \mathrm{~dB}$. Um paciente não foi capaz de realizar exames confiáveis após repetidas tentativas.

A administração de medicação ocular hipotensora diminuiu significativamente a PIO de $26,57 \pm 4,23 \mathrm{mmHg}$ (variando de 21 a $38 \mathrm{mmHg}$ ) para 16,54 $\pm 2,92 \mathrm{mmHg}$ (variando de 12 a $24 \mathrm{mmHg}$ ( $(\mathrm{p}=0,009)$, o que representou uma redução média de PIO de 37,80\%. Em 31 pacientes (84\%), uma medicação foi suficiente para produzir queda de $25 \%$ da PIO em relação ao valor inicial. Em 6 pacientes (16\%), mais de uma medicação foi necessária para produzir queda de $25 \%$ da PIO em relação ao valor inicial. Dos 37 pacientes incluídos no estudo, 27 (73\%) receberam somente análogos das prostaglandinas, $2(5 \%)$ receberam somente betabloqueadores, $5(14 \%)$ receberam associação fixa de análogo das prostaglandinas com betabloqueador e $3(8 \%)$ receberam betabloqueador mais análogo das prostaglandina em medicações separadas.

A tabela 1 revela que não houve diferença estatisticamente significativa entre os parâmetros do PVL medidos antes e após $a$ administração de medicação ocular hipotensora $(p>0,05)$.

\section{DISCUSS ̃̃O}

Como mencionado anteriormente, a avaliação topográfica do disco óptico e a análise da espessura da CFNR podem ser úteis no diagnóstico e no monitoramento de pacientes glaucomatosos. No entanto, é importante que estes exames não sofram influência de modificações da PIO, único parâmetro tratável em pacientes com glaucoma. Vários estudos sugerem que reduções da PIO após trabeculectomia resultam em alterações significativas dos parâmetros do disco óptico medidos com o HRT ou outros aparelhos que avaliam a topografia do disco óptico.

Foi realizada avaliação com a tomografia de varredura a laser (TVL) de 10 olhos de nove pacientes com glaucoma 3,7 meses após cirurgia filtrante e oito olhos de sete pacientes após 13,1 meses. Após 12,1 meses, uma redução da PIO de mais de $30 \%$ foi obtida em seis dos oito olhos, enquanto dois olhos obtiveram uma redução de menos de $15 \%$ apesar da introdução de medicação. Nos seis olhos com redução maior da PIO, o volume da escavação do disco óptico mostrou uma diminuição de mais de 30\%, enquanto nos dois olhos sem uma redução significativa da PIO houve um leve aumento do volume da escavação ${ }^{(12)}$.

Vinte e dois pacientes com vários tipos de glaucoma foram avaliados com a PVL antes e após a trabeculectomia. Todos os parâmetros examinados, exceto o volume da escavação, mostraram mudanças estatisticamente significantes após a cirurgia quando comparados aos valores pré-cirúrgicos. $\mathrm{O}$ aumento do volume da rima foi o parâmetro que mostrou modificação mais marcante, principalmente nos olhos com PIO pós-operatória menor do que $15 \mathrm{mmHg}^{(13)}$.

Imagens obtidas com o oftalmoscópio de varredura a laser (HRT) do olho de um paciente durante crise de glaucoma agudo (PIO máxima=60 $\mathrm{mmHg}$ ) e após resolução da mesma $(\mathrm{PIO}=$ $18 \mathrm{mmHg}$ ) num período de 24 horas, mostraram que após diminuição da PIO, houve redução significativa da área da escavação, volume da escavação, profundidade média da escavação, e profundidade máxima da escavação, e um aumento significativo da área da rima e da razão escavação/rima ${ }^{(14)}$.

Numa avaliação com o HRT 13 olhos de 13 pacientes glaucomatosos, nos quais houve uma redução de $25 \%$ ou mais na PIO dois meses pós-trabeculectomia, a razão escavação/área do disco reduziu significativamente, enquanto a área da rima e

\begin{tabular}{|lccc|}
\hline & \multicolumn{2}{c|}{ Tabela 1. Parâmetros da PVL antes e após redução da PIO } \\
& $\begin{array}{c}\text { Pré-medicação hipotensora } \\
\text { (média } \pm \text { desvio padrão) }\end{array}$ & $\begin{array}{c}\text { Pós-medicação hipotensora } \\
\text { (média } \pm \text { desvio padrão) }\end{array}$ & $\begin{array}{c}\text { Porcentagem de variação } \\
\text { do parâmetro (\%) }\end{array}$ \\
Integral polar total $\left(\mathrm{mm}^{2}\right)$ & $0,611 \pm 0,135$ & $0,603 \pm 0,127$ & $-1,36$ \\
Integral superior $\left(\mathrm{mm}^{2}\right)$ & $0,222 \pm 0,055$ & $0,218 \pm 0,051$ & $-1,91$ \\
Integral temporal $\left(\mathrm{mm}^{2}\right)$ & $0,059 \pm 0,017$ & $0,058 \pm 0,016$ & $-2,33$ \\
Integral inferior $\left(\mathrm{mm}^{2}\right)$ & $0,236 \pm 0,053$ & $0,235 \pm 0,052$ & $-0,23$ \\
Integral nasal $\left(\mathrm{mm}^{2}\right)$ & $0,094 \pm 0,024$ & $0,092 \pm 0,022$ & $-2,52$ \\
Média polar total $(\mu \mathrm{m})$ & $64,578 \pm 13,035$ & $64,576 \pm 13,940$ & 0,8500 \\
Média superior $(\mu \mathrm{m})$ & $71,143 \pm 16,066$ & $69,684 \pm 16,301$ & 0,00 \\
Média temporal $(\mu \mathrm{m})$ & $44,443 \pm 13,555$ & $44,865 \pm 13,371$ & 0,7713 \\
Média inferior $(\mu \mathrm{m})$ & $74,641 \pm 16,295$ & $74,522 \pm 17,843$ & 0,9638 \\
Média nasal $(\mu \mathrm{m})$ & $50,924 \pm 12,176$ & $51,005 \pm 11,836$ & 0,05 \\
\hline
\end{tabular}


o volume mostraram aumentos significativos. A área da rima neural, especialmente nas regiões súpero-temporal e ínferotemporal, mostrou aumento significativo. A diminuição na profundidade da escavação máxima correlacionou-se significativamente com a diminuição da $\mathrm{PIO}^{(15)}$.

Vinte e um pacientes glaucomatosos submetidos à trabeculectomia foram avaliados com o HRT. Análise quantitativa do disco óptico e perimetria computadorizada foram realizadas antes e após a cirurgia. Dezessete pacientes tiveram imagens pré e pós-operatórias válidas para análise. Onze dos 13 pacientes que tiveram uma redução da PIO de mais de $40 \%$ mostraram melhora nos parâmetros do disco óptico. Todos os quatro pacientes com uma redução da PIO menor que $25 \%$ mostraram piora na maioria dos parâmetros. Mudanças nos parâmetros do disco óptico foram altamente correlacionados com o percentual de redução da PIO e com a idade ${ }^{(16)}$.

Noventa e cinco pacientes submetidos à trabeculectomia foram avaliados com o HRT. As imagens foram obtidas no préoperatório e 3 meses, 1 ano e 2 anos após a cirurgia. Constatou-se um aumento significativo da área e do volume da rima 2 anos após a cirurgia ${ }^{(17)}$. Numa outra avaliação com o HRT de 25 olhos de 25 pacientes submetidos à trabeculectomia com imagens obtidas no pré-operatório e 2 semanas, 4 meses e 8 meses após a cirurgia, houve alterações no disco óptico detectadas 2 semanas após cirurgia, mas que não persistiram após 4 e 8 meses em olhos com glaucoma avançado, com exceção da forma da escavação ${ }^{(18)}$.

Outros estudos sugerem que reduções da PIO obtidas com tratamento medicamentoso também resultam em variação significativa das medidas da topografia do disco óptico. Dezessete adultos com glaucoma primário de ângulo aberto foram avaliados com o analisador de disco óptico de Rodenstock. As análises foram feitas em três momentos: a) com a PIO mais baixa na vigência de terapia (PIO 20,4 $\pm 2,5 \mathrm{mmHg}$ ); b) após elevação da PIO por falência terapêutica ou não adesão ao tratamento (PIO 31,1 $\pm 5,9 \mathrm{mmHg}$ ); c) após redução da PIO com terapia medicamentosa bem sucedida (PIO 19,3 $\pm 4,8 \mathrm{mmHg}$ ). A escavação do disco óptico aumentou significativamente após aumento da PIO e voltou ao padrão basal após redução consistente da $\mathrm{PIO}^{(19)}$

Vinte e nove pacientes com glaucoma ou hipertensão ocular foram avaliados com o HRT antes e após a administração de latanoprost. Nenhum parâmetro mudou significativamente após a redução da $\mathrm{PIO}^{(20)}$. No entanto, considerando-se somente os pacientes nos quais houve uma redução da PIO de mais de $25 \%$, quatro parâmetros mudaram significativamente: área da escavação, volume da escavação e razão escavação/ disco diminuíram, enquanto a área da rima aumentou.

De maneira geral, os estudos descritos acima revelam, como resultado da diminuição da PIO, redução do volume e área de escavação e aumento do volume de rima. Estas modificações são compatíveis com um deslocamento anterior da lâmina crivosa pós-redução da PIO, que resulta em alteração dos parâmetros avaliados.
Poucos estudos avaliaram as alterações das medidas da espessura da CFNR após redução da PIO em pacientes glaucomatosos. Numa avaliação com a PVL de 46 olhos de 46 pacientes com glaucoma primário de ângulo aberto, nos quais a PIO foi reduzida em mais de $30 \%$ pós-trabeculectomia, as imagens foram obtidas no pré-operatório e 3 e 6 meses após o procedimento cirúrgico. A espessura da CFNR nas regiões súpero-temporal e ínfero-temporal foi significativamente maior no pós-operatório do que no pré, principalmente nos casos de glaucoma inicial com MD não muito diminuído ${ }^{(21)}$.

Não há, no entanto, estudos que avaliem os efeitos da redução da PIO obtida com tratamento medicamentoso sobre as medidas do PVL. Ao contrário do estudo citado anteriormente no qual a PIO foi reduzida cirurgicamente, não observamos alterações dos parâmetros do PVL após redução de $38 \%$ da PIO. De maneira semelhante ao estudo supracitado, a maioria dos pacientes apresentava $\mathrm{MD}>-6 \mathrm{~dB}$. Portanto, não podemos justificar a ausência de modificações na CFNR pela inclusão de casos preponderantemente severos, nos quais a possibilidade de alterações na CFNR é limitada pela escassez de fibras. No entanto, nossa casuística incluiu pacientes hipertensos oculares e glaucomatosos, ao contrário do estudo supracitado, que incluiu apenas pacientes com glaucoma primário de ângulo aberto. Outra hipótese, menos provável, é de que os efeitos da diminuição da PIO ocorram após certo período de tempo. No nosso estudo, as imagens foram obtidas logo após a redução da PIO (entre 15 e 45 dias), um intervalo pequeno quando comparado ao outro estudo, que realizou novo exame 3 a 6 meses após a cirurgia. No entanto, a hipótese mais provável que justifica as diferenças entre os dois estudos está relacionada aos níveis de PIO obtidos com o tratamento medicamentoso $(16,54 \pm 2,92 \mathrm{mmHg}$ no nosso estudo) e cirúrgico $\left(10,2 \pm 3,7 \mathrm{mmHg}\right.$ no outro estudo ${ }^{(21)}$.

Assim, concluímos que a redução da PIO com o uso de medicação ocular hipotensora não altera a medida da espessura da CFNR pela PVL em pacientes com glaucoma ou hipertensão ocular. Isto sugere que esta tecnologia possa ser empregada no acompanhamento de pacientes com glaucoma ou hipertensão ocular mesmo após redução da PIO obtida com medicamentos.

\section{ABSTRACT}

Purpose: To evaluate changes in retinal nerve fiber layer thickness as measured by scanning laser polarimetry (SLP) after the use of medication to reduce intraocular pressure (IOP) in glaucomatous or ocular hypertensive patients. Methods: The authors prospectively enrolled 37 eyes of 37 patients in whom IOP was reduced by more than $25 \%$ after the use of medication. The images were obtained before and 15 to 30 days after the introduction of medication. The SLP parameters measured before and after the use of medication were compared using paired Student's t Test. Results: The mean IOP was significantly reduced from $26.57 \pm 4.23 \mathrm{mmHg}$ to 16.54 
$\pm 2.92 \mathrm{mmHg}$ after the use of medication $(\mathrm{p}<0.05)$. None of the 10 SLP analyzed parameters was significantly affected by the reduction of IOP with medication $(\mathrm{p}>0.05)$. Conclusion: The retinal nerve fiber layer thickness, as measured by SLP, is not affected by the reduction of IOP with medication in patients with glaucoma or ocular hypertension.

Keywords: Glaucoma; Intraocular pressure/drug therapy; Nerve fibers/pathology; Retina/pathology; Diagnostic techniques, ophthalmological; Lasers/diagnostic use

\section{REFERÊNCIAS}

1. Glaucoma Primário de Ângulo Aberto: $1^{\circ}$ Consenso da Sociedade Brasileira de Glaucoma. São Paulo: BG Cultural; 2001. v.1. 54p.

2. Quigley HA, Addicks EM, Green WR. Optic nerve damage in human glaucoma. III. Quantitative correlation of nerve fiber loss and visual field defect in glaucoma, ischemic neuropathy, papilledema, and toxic neuropathy. Arch Ophthalmol. 1982;100(1):135-46.

3. Kahn HA, Leibowitz H, Ganley JP, Kini M, Colton T, Nickerson R, Dawber TR. Randomized controlled clinical trial. National Eye Institute workshop for ophthalmologists. Standardizing diagnostic procedures. Am J Ophthalmol. 1975;79(5):768-75.

4. Lichter PR. Variability of expert observers in evaluating the optic disc. Trans Am Ophthalmol Soc. 1976;74:532-72.

5. Weinreb RN, Dreher AW, Coleman A, Quigley H, Shaw B Reiter K. Histopathologic validation of Fourier-ellipsometry measurements of optic nerve topography in glaucoma. Invest Ophthalmol Vis Sci 1998;29:1294-8.

6. Kamal DS, Bunce C, Hitchings RA. Use of the GDx to detect differences in retinal nerve fibre layer thickness between normal, ocular hypertensive and early glaucomatous eyes. Eye. 2000;14(Pt 3A):367-70.

7. Hollo G, Szabo A, Vargha P. Scanning laser polarimetry versus frequency doubling perimetry and conventional threshold perimetry: changes during a 12month follow-up in preperimetric glaucoma. A pilot study. Acta Ophthalmol Scand. 2001;79(4):403-7.

8. Tezel G, Siegmund KD, Trinkaus K, Wax MB, Kass MA, Kolker AE. Clini- cal factors associated with progression of glaucomatous optic disc damage in treated patients. Arch Ophthalmol. 2001;119(6):813-8.

9. Bowd C, Weinreb RN, Lee B, Emdadi A, Zangwill LM. Optic disk topography after medical treatment to reduce intraocular pressure. Am J Ophthalmol. 2000;130(3):280-6.

10. Chylack LT Jr, Wolfe JK, Singer DM, Leske MC, Bullimore MA, Bailey IL, et al. The Lens Opacities Classification System III. The Longitudinal Study of Cataract Study Group. Arch Ophthalmol. 1993;111(6):831-6.

11. Zangwill LM, Bowd C, Berry CC, Williams J, Blumenthal EZ, SanchezGaleana CA, et al. Discriminating between normal and glaucomatous eyes using the Heidelberg Retina Tomograph, GDx Nerve Fiber Analyzer, and Optical Coherence Tomograph. Arch Ophthalmol. 2001;119(7):985-93.

12. Raitta C, Tomita G, Vesti E, Harju M, Nakao H. Optic disc topography before and after trabeculectomy in advanced glaucoma. Ophthalmic Surg Lasers. 1996; 27(5):349-54.

13. Yoshikawa K, Inoue Y. Changes in optic disc parameters after intraocular pressure reduction in adult glaucoma patients. Jpn J Ophthalmol. 1999;43(3): 225-31.

14. Lee BL, Zangwill L, Weinreb RB. Change in optic disc topography associated with diurnal variation in intraocular pressure. J Glaucoma. 1999;8(3):221-3.

15. Park KH, Kim DM, Youn DH. Short-term change of optic nerve head topography after trabeculectomy in adult glaucoma patients as measured by Heidelberg retina tomograph. Korean J Ophthalmol. 1997;11(1):1-6.

16. Lesk MR, Spaeth GL, Azuara-Blanco A, Araujo SV, Katz LJ, Terebuh AK, et al. Reversal of optic disc cupping after glaucoma surgery analyzed with a scanning laser tomograph. Ophthalmology. 1999;106(5):1013-8.

17. Kotecha A, Siriwardena D, Fitzke FW, Hitchings RA, Khaw PT. Optic disc changes following trabeculectomy: longitudinal and localization of change. $\mathrm{Br} \mathrm{J}$ Ophthalmol. 2001;85(8):956-61.

18. Topouzis F, Peng F, Kotas-Neumann R, Garcia R, Sanguinet J, Yu F, Coleman AL. Longitudinal changes in the optic disc topography of adult patients after trabeculectomy. Ophthalmology. 1999;106(6):1147-51.

19. Parrow KA, Shin DH, Tsai CS, Hong YJ, Juzych MS, Shi DX. Intraocular pressure-dependent dynamic changes of optic disc cupping in adult glaucoma patients. Ophthalmology. 1992;99(1):36-40.

20. Bowd C, Weinreb RN, Lee B, Emdadi A, Zangwill LM. Optic disk topography after medical treatment to reduce intraocular pressure. Am J Ophthalmol. 2000;130(3):280-6

21. Yamada N, Tomita G, Yamamoto T, Kitazawa Y. Changes in the nerve fiber layer thickness following a reduction of intraocular pressure after trabeculectomy. J Glaucoma. 2000;9(5):371-5. 